\title{
LE APPLICAZIONI GEODETICHE DEL RADAR
}

\author{
GHOVANXI BOAGA
}

Il prof. C. A llant dell'Univer-ila di Londra in occatione del.

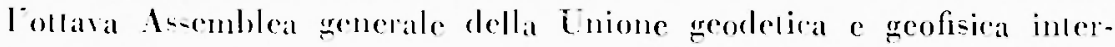
nazionale lenula-i nello seoro agoslo ad Oslo, presentò un inleressinte Rapporto walle applicazioni weodeliche della racliotelerrafia con risuardo specialmente a quelle di lipo Radar.

Il romandiante E. Bergatrand in late oreatione ha pure riferibo -ulle mi-ure di di-lanze con procedimenti di allal frequenza esenuite in Datnimatrat ed il prof. Th. Ver-telle del Reale ufferio idrogratieo olanlese rileri sul principio del Derea.

Recentemente il prof. Ilarl ha pubblitialo un interesante tudio. - cuppe -11 que-ti argomenti, - -ul "Bubletin Gededeiguen (n. 10, 19)18).

Que-1i nuosi preadinenli -one: drelinali a portare prandi contribuli alle mi-urazioni geodeliche. !e quali abbuncicinetanno yuati complelamenwe le mi-ure angolati ed incrementeranno sempere piii puelle di lunglue di-lanze. Si i a!l’nizio percio di un nolerele movimento che invele gran piate. non solo dellit weode-ia opelalliva, ma alterei dellat geode-ial reorelica per a guanlo conierne le leorie della mi-ula e delle compensazioni. le 'fuali ultime dorramno pow wariar-i non lanto - rome so stallo fallo finora -ulla triangolazione (ni-ure di una o piì bati c di angroli), ma ben-i -ulla trilaterasione (misure di sole disianze lincani). Sollo yueso punto di visia erli argomenli di eni i $i$ lallo

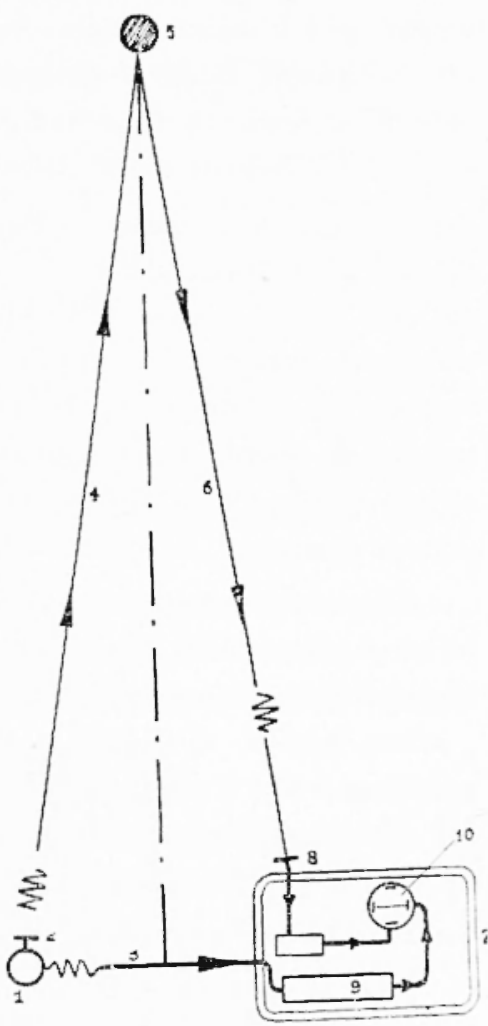

Piv. 1 - Scliema del funzionamento del radio-localizzatore 
renno aldqui-lano grande importamzal sperialmente per i rollegannenti di isole al rontinenti e di continenti lra loro, potendo-i ora mi-lualle

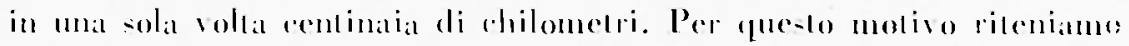
ulile dapprima illu-lrare brevemente i primeipî sui quali si hatano i vari procedimenti di misurazone delle di-lanze ron meza elentridi. poi mettere in rilievo i ri-mlati di alemi lasori sperimentali anewati

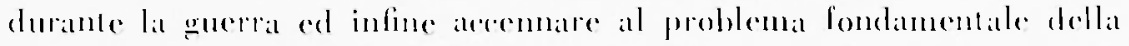
compen-azione della trilaterizione, ron lapporto pure di qualethe ronlribute originale.

1. - Il prineipio -ul quale - appogeniano que-li prosedimenti is quello della eco (riflesione) delle ratloonde. quando vengono lam ialle

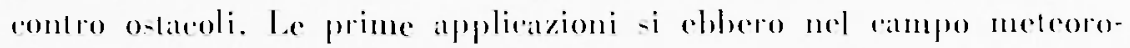

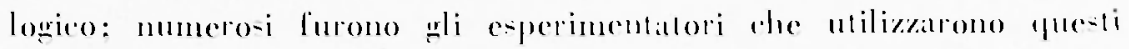
melodi per le mi-ne delle alleze di dillerenti -liali di aria ionizalli.

Lat parolat Radar i formala con la primat sillabla della prima pat-

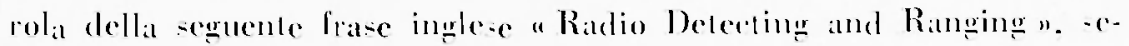
fruila dalle iniziali delle allere tre patrole.

Il radio-localizatore (Radar) i eo-tituito (vedi to -rhemil nell:t

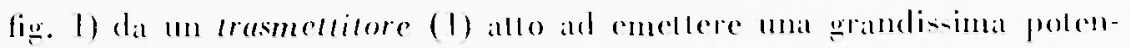
za, per mal frazione dellordine di milione-imi di -eromdo. La trasmis-ione awiene ad inpulsi dellat durala di reira 11 milione-imi di serondo ed is interealiala dis ma pall-al - periodo di -ilenzio - relat-

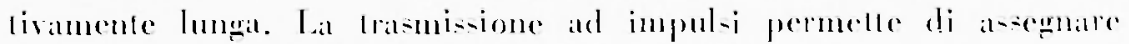
all impianto piecole dimensioni in modo rhe eno puo enere fiucilmente. atulotra-portalo.

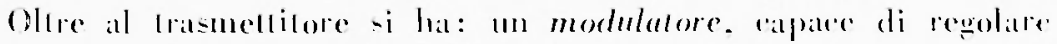
le tra-missioni: mal antenna a rifletlore orientabi!e (2) rhe irratia gili impulsi nella direzione volutat - 11 piano orizzontale rondotes per il punto stazione. in modo dis raggiungere loosanolo (5). mediante lit

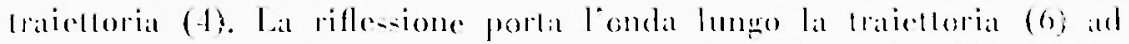
un ricevilore (8) e ad un mbo a raggi ralodici (101). - - rui -i lammo

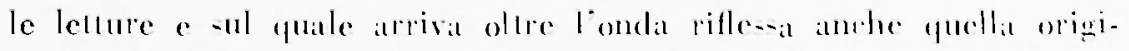
natria, obbligata a pasalre per loilinerario (i). (9). (redi figg. 1).

Per comprendere il funzionamento di questo lubo. denominato il P.P.I. rice indiratore del piano di posizione, i eon-ideri mal valsola

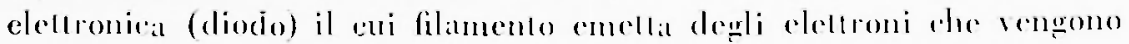




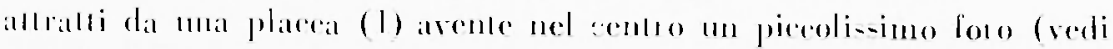
liw. 2).

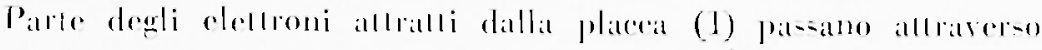

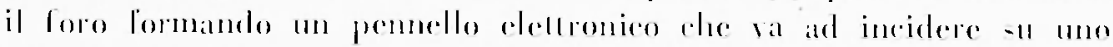

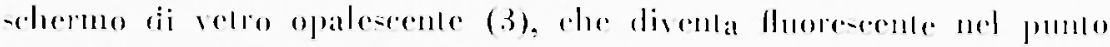

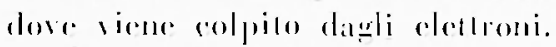

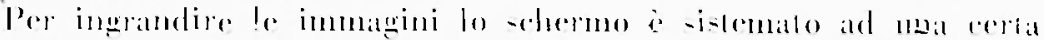
distamzar dalla placeat. collowando fra 10 -rlecrumo a la placea

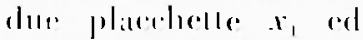
r. del lipos di quall. m-ille ne.i condentallori in moslo elece il liareio eleillonico pais nello -pazio rompreso dalle

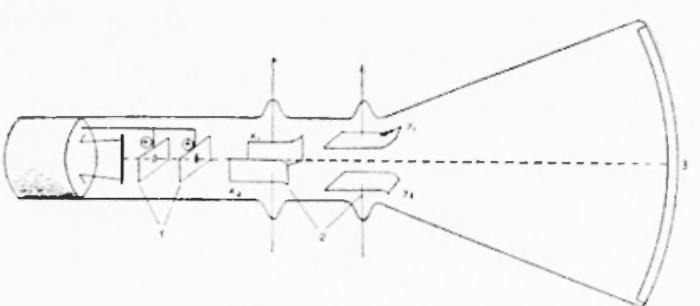

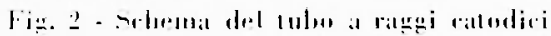

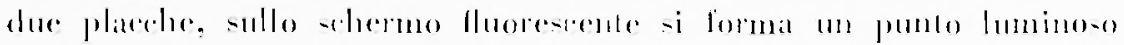

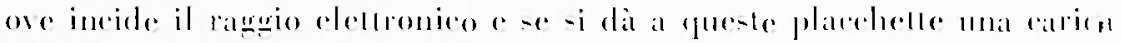

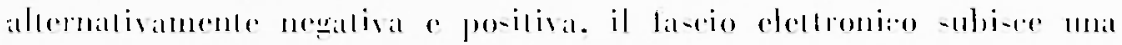

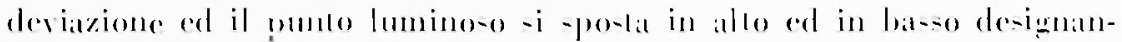

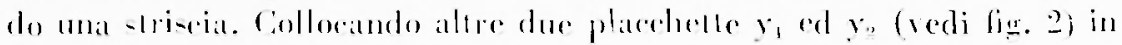
direzioni normali alle prime, il punto lumino-o -i posterit in som-o

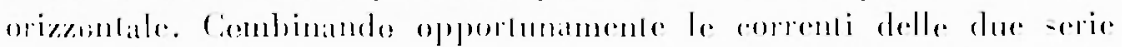

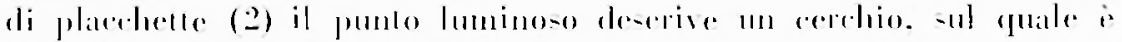

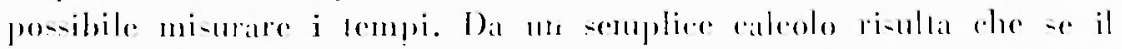

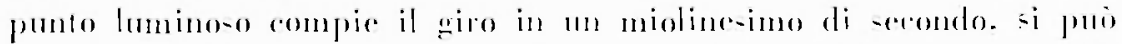
arrivare a determinare lajprowimasione ehe -i puie ollenere nelle mi-ure di di-lanze.

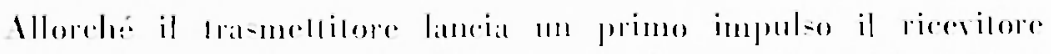

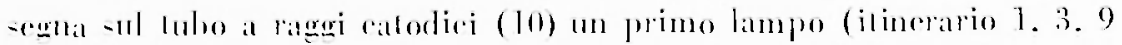

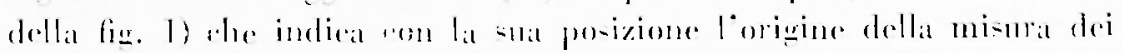

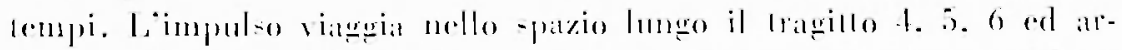

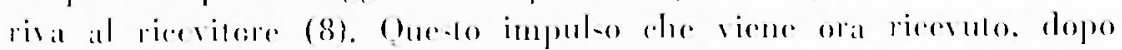

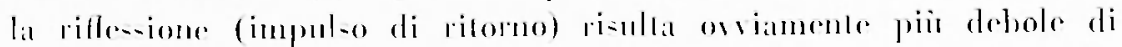

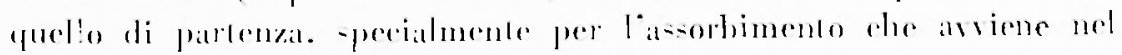

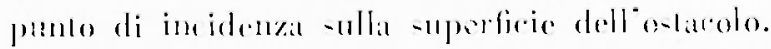

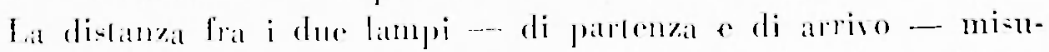

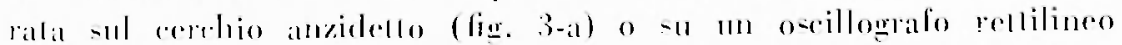

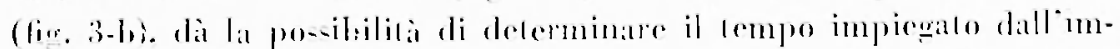

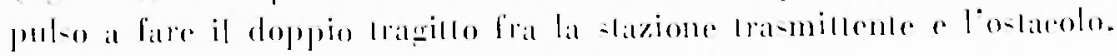


visibile dalla tazione, e conseguentemente, poiche la velocita delle radioonde nell'aria roincide a tulli ghi effelli plalici ron quella della luce nel ruoto, si può risarare la distanza fra la slazione clo-lacolo c ciò con ma approssimazione che dipende micamente dalla preci-

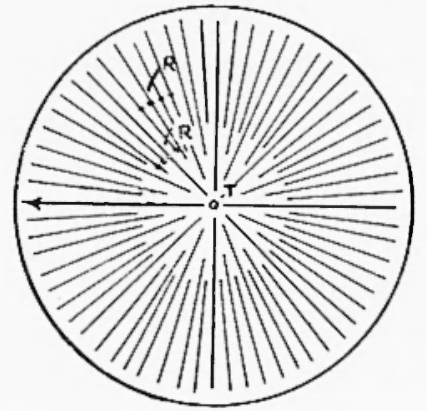

Fig. 3 il - P.P.I.; $T=$ impulso di trasmissione (itinerario 1 , 3,10 della fig. 1); $\mathrm{R}=$ impulso di riflessione $(1,2,4,5,6$, 8,10 , dellat fig. li sione della misura del cempo. Poirlé lat velocilà delle radioonde è dell'ordine di $300 \mathrm{~m}$ in un milione-imo di secondo, grli intervilli di lempo che si derono misurare sono molto piecoli, anele per lunglae dislanze, ma lat ternilat elettronical odiema pos-iede dei mezzi tali, dis poter romodamente effetluate lali mi-ure, risultando wi praliamente privi di inerzial si puó arrivare a rompulare senzal diffeolla tempuscoli dell'ordine del rentomilionesimo del secondo $\left(1 \times 10^{-8}\right.$ sec $)$.

Da quanto precede ri-ulta chiaro whe le radioonde cone-es dal tratsmeltitore del Radar debbono essere ald altiwimal frequenzal per date modo all'antemia di concentrarle in un fascio as-ai tretlo (c.fr. G. Simeon. "Progresi nei metodi dellat navigazione" in tmali dell Ist. Univ. Navale, 1947.

I) complesso strumentale descritto può denominarsi anche radioecometro o meglio radiotelemetro.

La portata pratilia del radiolelemetro è influcnzatia però da diver-

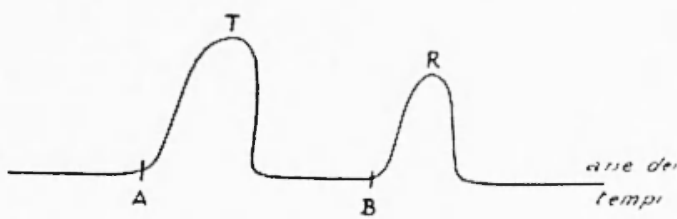

Fig. 3 b $-\mathrm{T}=$ impulso di tratsisione; $\mathrm{R}=\mathrm{im}$ pulso di rilles:ione; $B-A=$ intervallo di tempo durante il quale limpulso hat compiuto il viagqio di andatia e ritomo dalla stazione al segrate

si faltori di earallere fisico, quali: quota di in-lallazione, carallerisliche elettriche del mezzo interpo-lo, dimen-ioni e natura dello-kacolo, orientamento delle antenne; potenza e sensibilita dell appraratu. interferenza di onde riflese dalla ionosfera, reflazione dell arial. Se S è la sezione di un ostacolo insallalo entro il raggio deflorizzonle visibile dalla stazione, $P$ la potenza del Radar, d la durata dell impuloo, $G$ il guadlagno dell'intennia, $K^{\prime}$ e $K^{\prime \prime}$ due coefficienti alli a tenere conto delle caratteristiche del romplesso strumentale, quali allitudine del- 


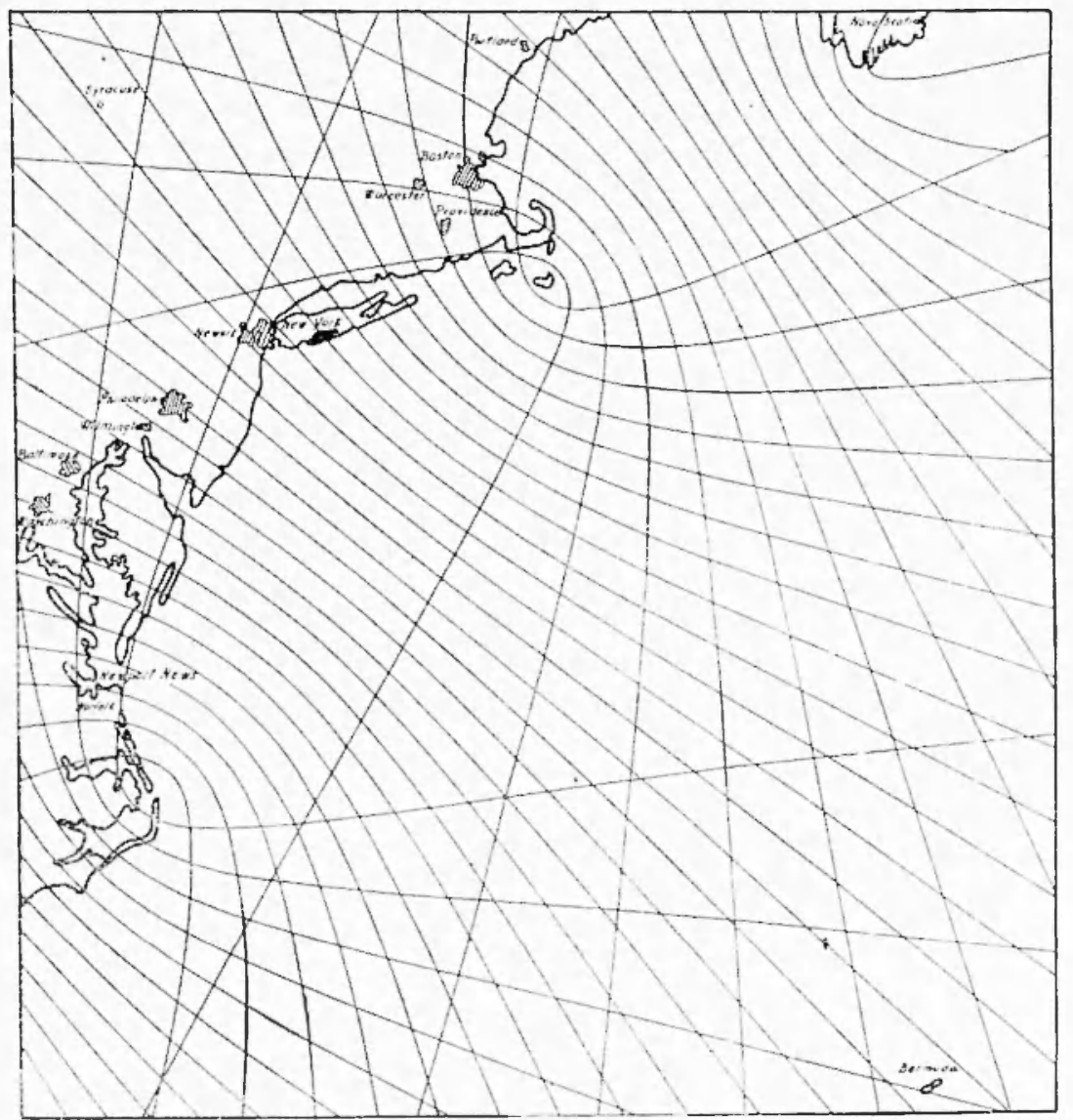

Fig. 1. Famiglie di iperboli generate da una doppia hase di Loran ifigura rilevata dalla Nota del prof. Lombardinil

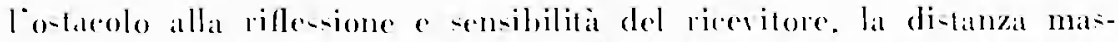
-iuna $\left(D_{\operatorname{mav}}\right)$ che puo essere rilevata. i fornita dalla seguente formula:

$$
D_{\operatorname{mnx}}=\sqrt[t]{\frac{K^{\prime}}{K^{\prime \prime}} \cdot P \cdot G^{2} \cdot S \cdot d}
$$

(afi. P. Lombaldini. "Propettive di una radiotopografial ". Rivi=ta loniterso. 1918).

Lepperienza ha dimostrato poi rhe per o-lacoli alti al snolo possono servire onde relativamente lunghe, per oflacoli basi (-ul smolo) onde piì hovi. 
Medianle tramisioni di impulei brevi (p. r. di $\left.15: 10^{-\mathrm{s}} \mathrm{sec}\right)$ -i ollengeno nelle distanze presi-joni di rirea $10 \mathrm{~m}$.

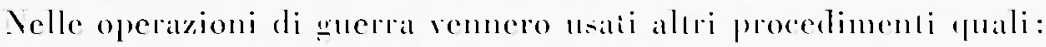
il Redar a rispusta (Beateon), dispo-ilivo ehe permelle di edestuate

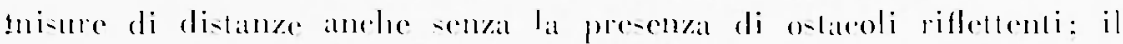

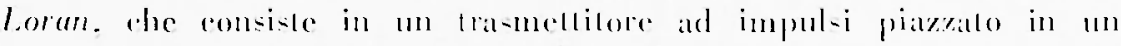

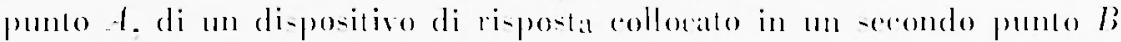
c di un ricevilore Radar. il quale o installato in lun ler\%o punto $\mathrm{Y}$ di

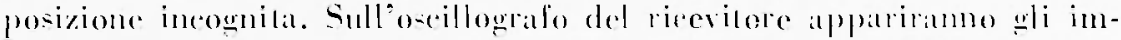
pulsi di te di $B$ : il loro reciproco ritardo $t$ is fornilo dallat:

$$
t=\frac{A B \cdot \overline{B X}}{c} \cdot \frac{A X}{c}=\frac{\overline{B X}-\bar{A}}{c}+\frac{A B}{c}
$$

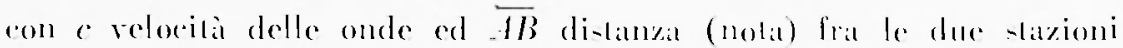

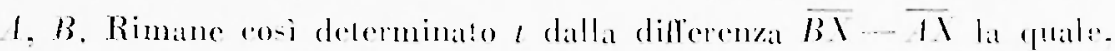

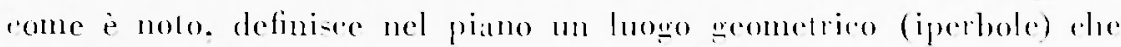

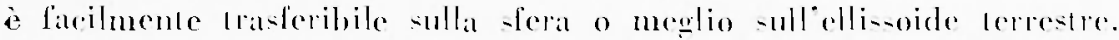

Disponendo di mar eoppia di hatei si hat la pos-ibilila di indivi-

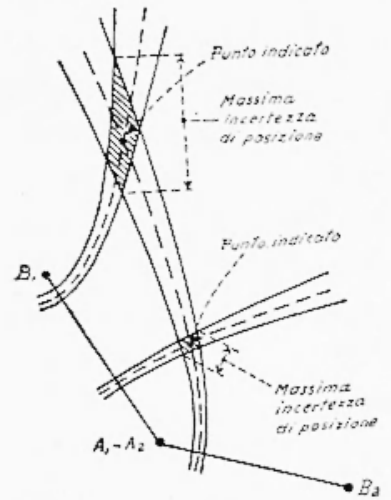

Fir. j. Zuna di errore nella determinazione col metodo iperbolieo figura rilevata dal. la Nota del prof. Lombardini

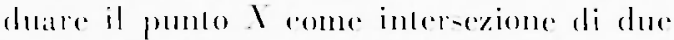

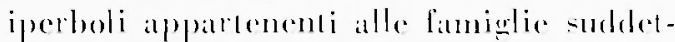
te (redi fier. 4 a lig. i).

Il melodo lorran. il rui nome provicene da "long Ramg Javigationn a che negeli ambicnti ingle-i i romo-eiuto rol noms

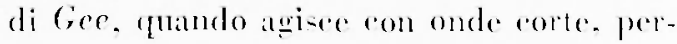
melle di esendere l’impiegro del Radal a di-lanze di mole centinaia di rhilometri. Sceondo I. I. Pierese (An introduretion lo Lotan: 19-16) le di-tanze ron pue-to procedimento si posenno ottenere con erroti medi di $+3 \mathrm{~m}$.

Sono sali esogeitali anche celememi ad interferenza. nta owgigiorno precontano

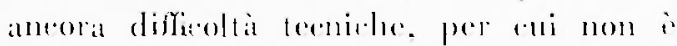
posibile allualmente fare delle applicizioni propriamente weodelidele.

Una ipplieazione notevole del metodo delle interferenze is dala dal -islema Decea, duale del sisemal Lomene diamzi firerdato. Amele eol

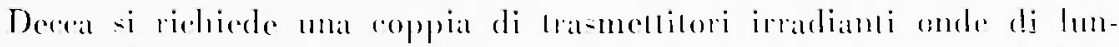
gheze ohe tamno in rapporti smpliei ed installati in due punti $A$ 


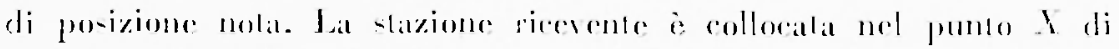

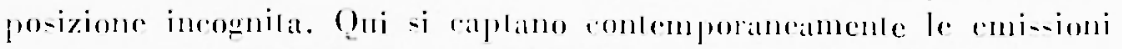

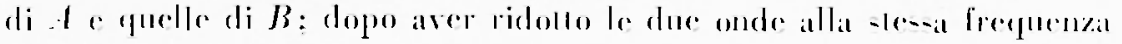
-i confrontano le fasi con un fa-ometro. l.e delerminazioni ro-i -i riferi-eono ad mal differenza di di-lanze; anelue qui dumpue -i definisere

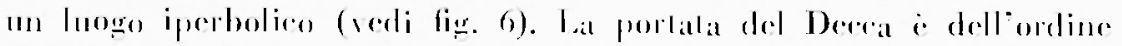

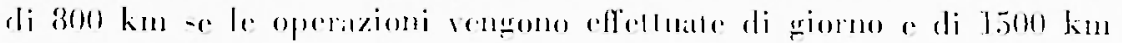
-e reflellualle di nolte.

In uleriore telemetro is hatalo -ulla curiazione di frequenza: es-ol

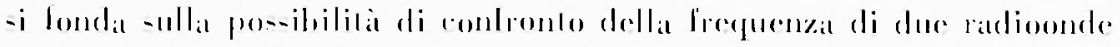

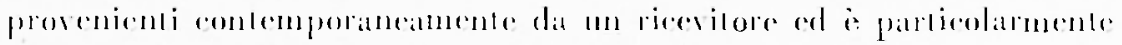

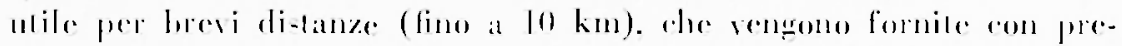
ri-ioni inferiori al melro.

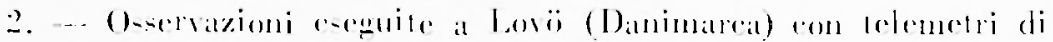

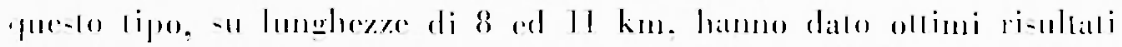

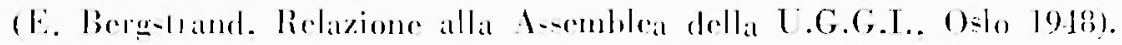

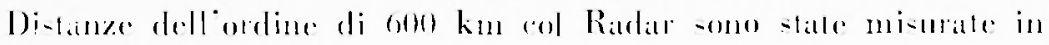
Italia nel 1955 da operatori ingle-i, ollenendo pure lumoni ri-ulanli.

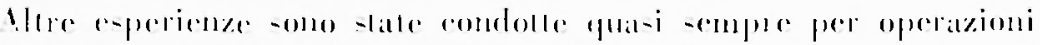

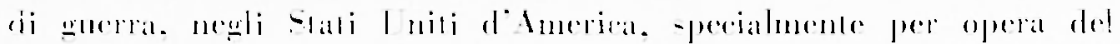

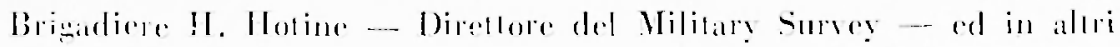
l'ile-i.

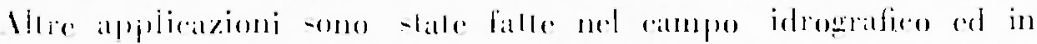

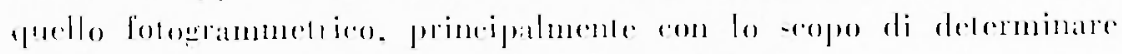

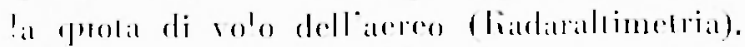

Nhri -i-temi sono quelli rhe sanno -ollo i nomi: Oboc e Shoran.

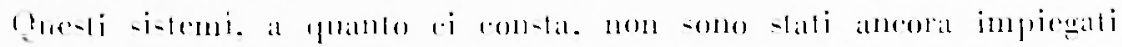

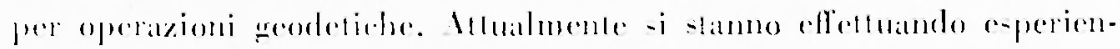

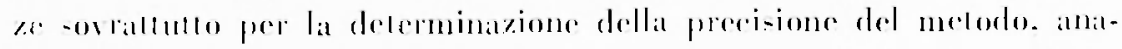
lizzando be varic rall-e di cromi.

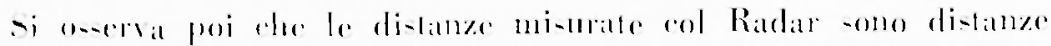

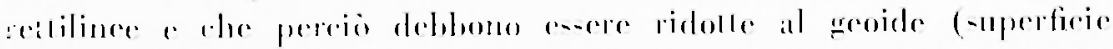

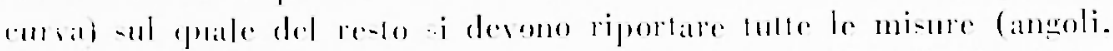

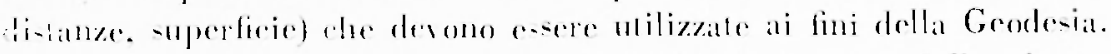

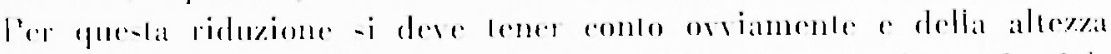

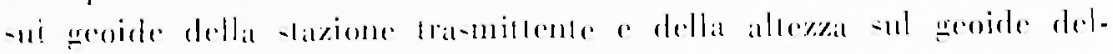

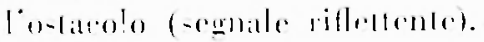




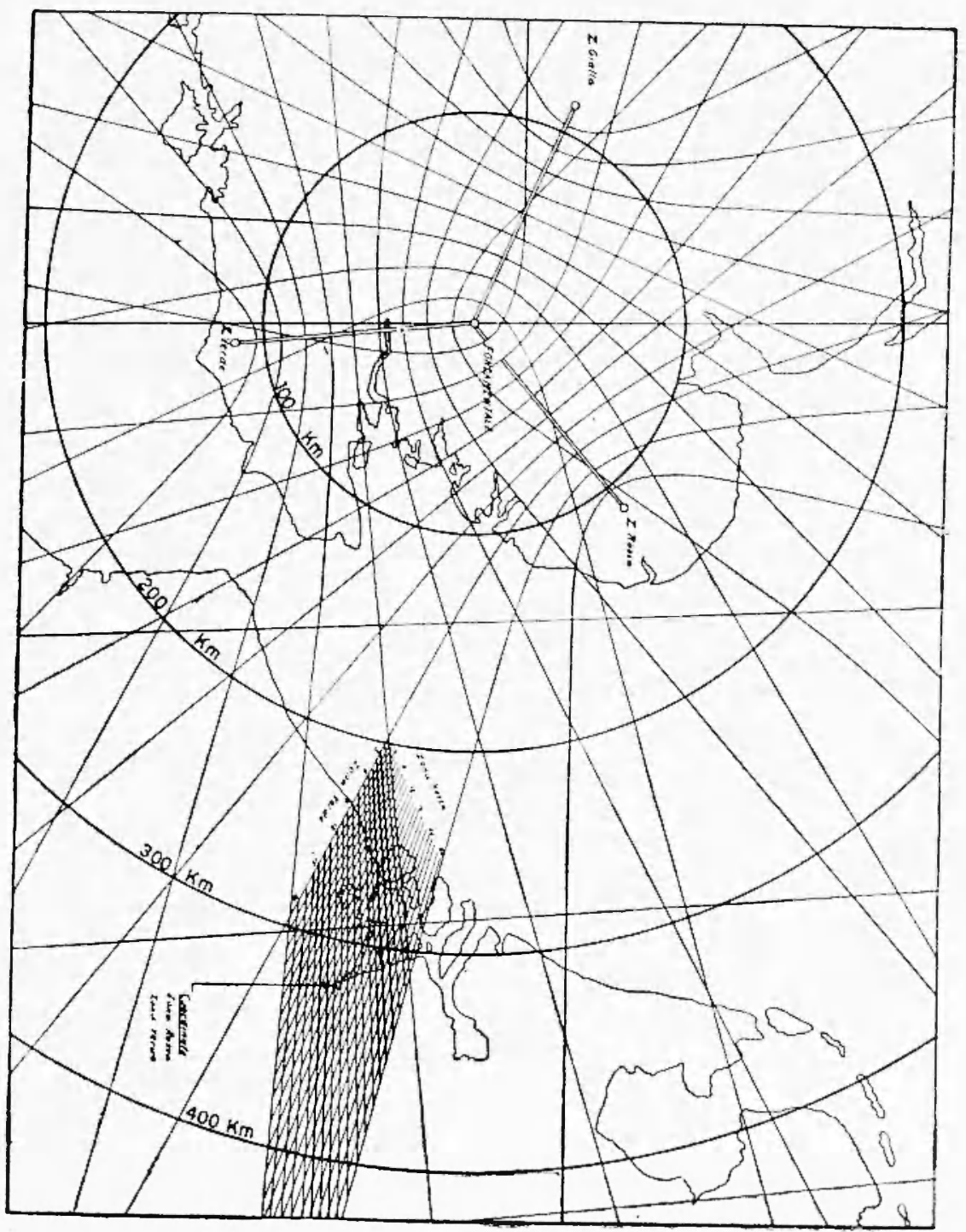

Fig. 6 - Famiglie di iperboli generate da tre basi Decea (schiave). La stazione

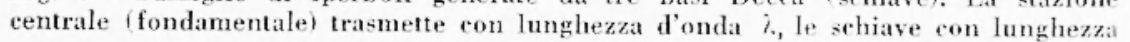
d'onda $\frac{\mathrm{m}}{\mathrm{n}} \hat{\lambda}$. 
La velocita $c$ delle onde elettromanneliche nello spazio is legatia all’indice di rifrazione atmo-ferico $l 6$ ed alla relocita $l$ di propatatzione della luee, dalla relaxione:

$$
c=\mu . V
$$

L'indice $l$, che a variabile con le condizioni atmoferiche, i può ricavatre dalla seguente formula di Englund-Crawford-Mumford:

$$
\left.(11-1) \times 10^{\prime}=\frac{p}{2 T}\left\{2,11+\frac{100 w}{p}\left(\frac{101,59}{T}-0,00293\right)\right\}\right\}
$$

in funzione dei dati meteorologici: $T$ temperatma asooluta (centigra(lis), presione atmo-feriea copresal in millimetri di mereurio, $u$ lat variazione di presione dovuta alla premenzal del vapor d'aregua e pure epresea in millimetri di mereurio.

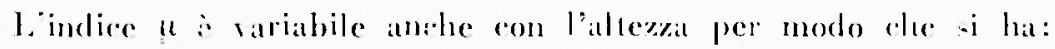

$$
u_{h}=u_{n}(1-h . k)
$$

con evidente innifiealo degli indiri appoti a 41 : il fatlore $K$ vale approsinalivamente $1 / t R$ con $R$ ratgrgio medio della Terral ed $h$ allezar dellat tiazione.

Secondo atcorale mi-urazioni compiute da Sunilh, Franklin, Whiting nel 1917 nelle l-ole ingle-i, a varie alteme, la relocila $I$ varia nel erwuente modo:

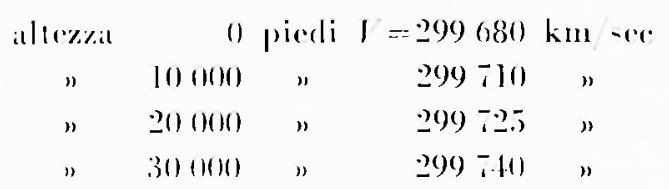

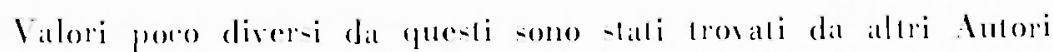
(Jone, Conford, anno 1918) in altre regioni.

In partieglare poi nel 1935 Michel-on-Pearec-Pearson hamo oltenulo il valore (299 7.t 11$) \mathrm{km}$-ece (cfr. "Velocity of Light in a Parlial Villumm n, Lar. Journ. Vol. 82): Anderson nel 19-1 con metodi

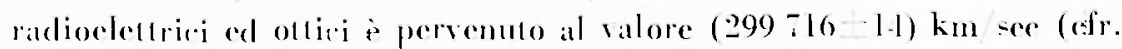
"Final Mean-urement of the Velocily of Light w. Journ. po. Opt. Sor.

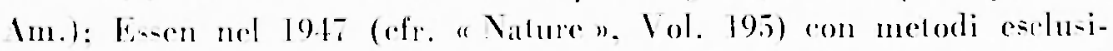
vamente elettriti ha trovalo un valore un po" maggiore dei precedenti

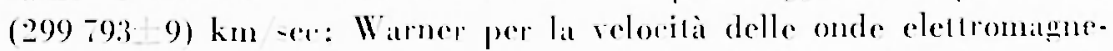

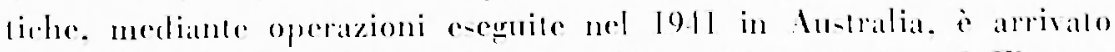
a tabiline il valore $29976 \mathrm{~km}$-ce (cfr. "The velocily of Electro-

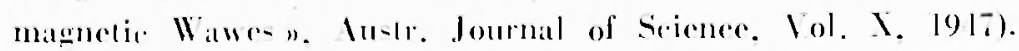




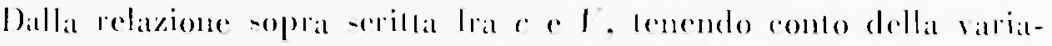

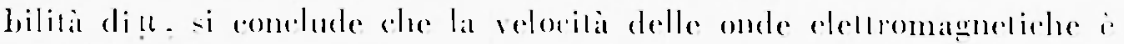
varialile eol mulare delle condizioni almo-feriehe: inollere puando le

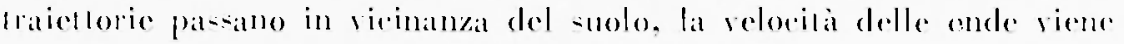

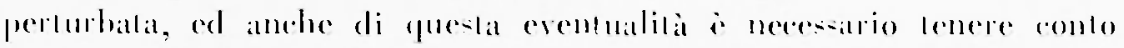
nelle applitazioni geodeliche.

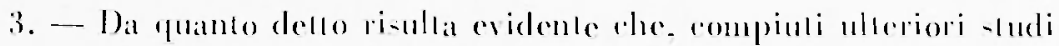

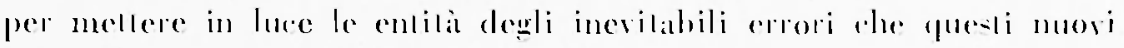

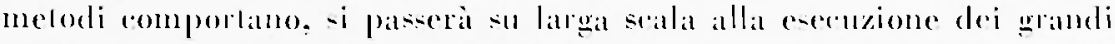
collegamenti geodelide in modo da unire le lriangolazioni e-i-lenti -nlle isole a srandi di-lanze dai rentinenli, ron quelle -labilile slli continenli sle-i. Le misure lineari ehe si ollerramo dostanno allora esere arog-

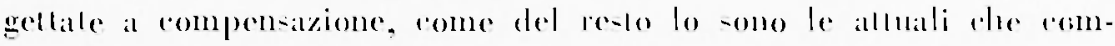
prendomo angoli e disimze.

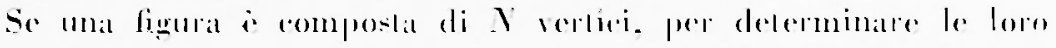

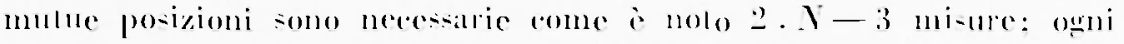

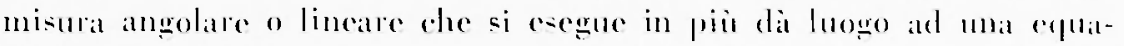

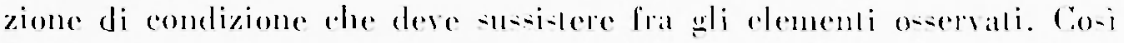

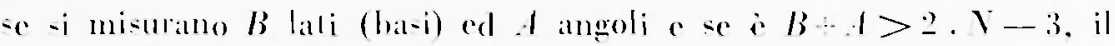
numero Ee delle equazioni di rondizione ir dalo dalla:

$$
I,=1-2,13
$$

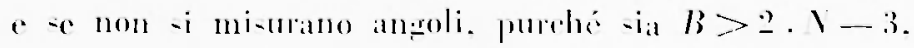

$$
E .=B-2.1-3 .
$$

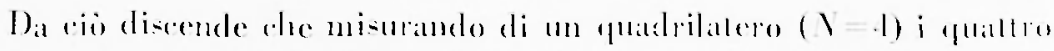

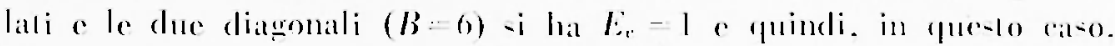

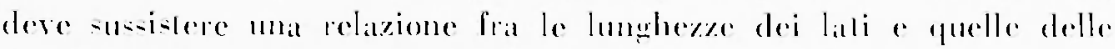
diaıronali.

Generalizzando la fue-lione si lrovano ri-nllali inleremanti eon-iderando p.e. un pentagono $(\mathrm{V}-5)$ e mi-mbando i lali e le diagonali

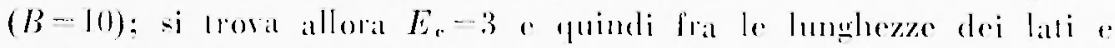
quelle delle diagonali di un pentagono dehbono sls-isere tre relazioni fra loro indipendenti.

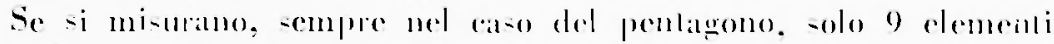

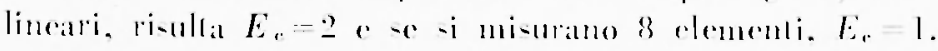

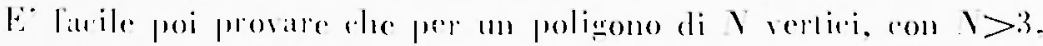
misurando lulli i lali e lulle le diagronali, il muncero di equatrioni di 


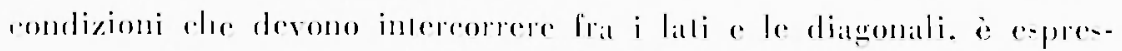
-1) (ial! Iil:

$$
E_{v}=\frac{1}{2}(N-2)(N-3)
$$

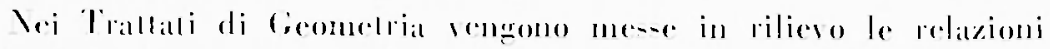

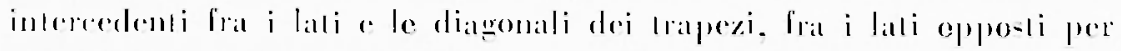

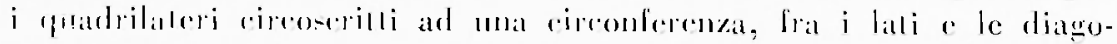

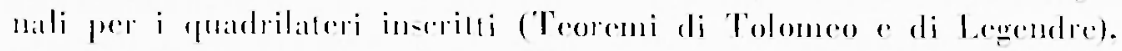

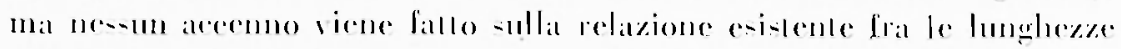

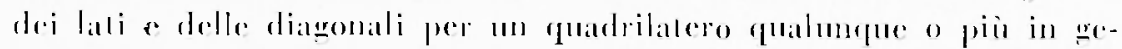
serale frat yli elementi, pure lineari - lali e diagenali - di un polizono irregolare fual-iat-i. Pero greonelri rommi -i -ono oreupali di

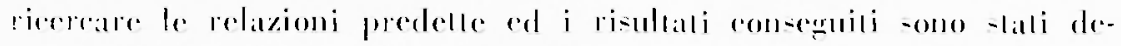

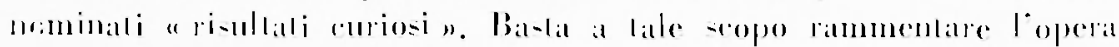

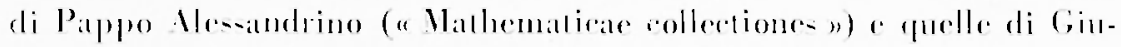

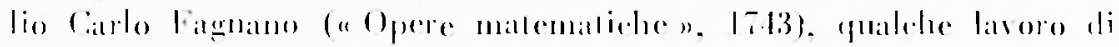

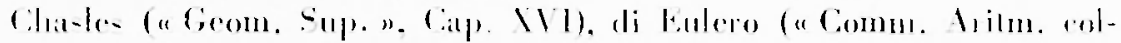
leriaren. 1819). ere.

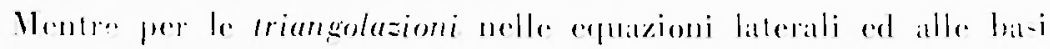
predomina il "Teoremal dei seni ". per le rrilaterazioni nelle formule

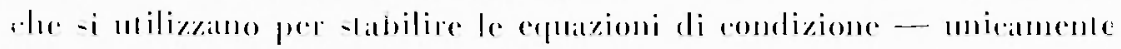

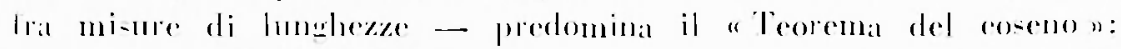

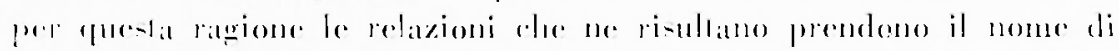
cidentili di Carnoln".

Rerentemente F. Warrlalow-ki (War-alsia. 1918) in mal Nola dal

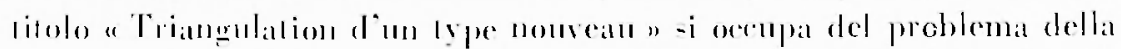
compen-azione della trilaterazione, ma molto prima (1935) lo serivente. in mol Jota in-erila nella Rivisa del Cala-lo a dej SS.TT.FE. dal

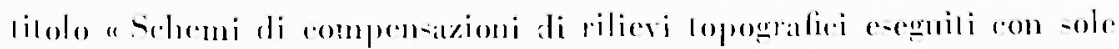
ani-ure linearin e poi riportala net proprio "Trallato di Geodesia e

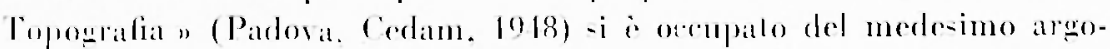

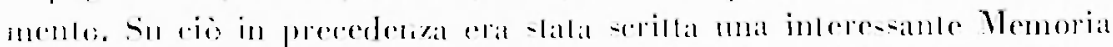

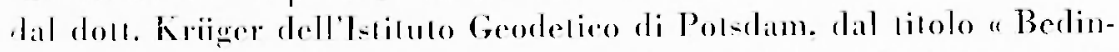

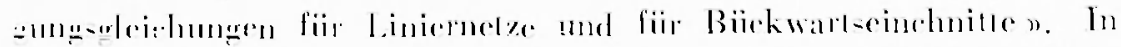

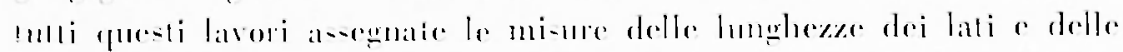

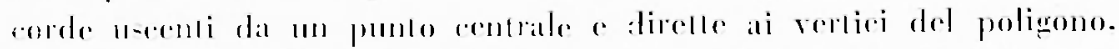

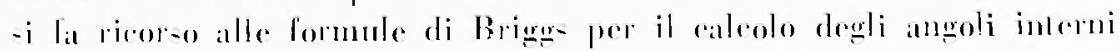

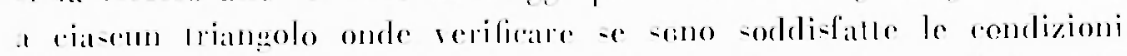
he rapprenentano i legami geomelidei delle fegure. Tn particolare la 
somma degli angoli attorno al giro di orizzonte del punto centrale deve risultare di quattro angoli relti; se ciò non accade si correggono le lunghezze delle misure provenienti dalle osservazioni ricorrendo alles relazioni che derivano da quelle esprimenti il Teorema di Carnol, dopo di averle diflerenziate, considerando variabili lutti i paramelri in esse contenuli (vedi p. es. G. Boagal, luogo ditalo). Poiche le variazioni dei lati risultano cosi funzioni di quelle degli angoli attorno al nodo (punto centrale dianzi ronsiderato), cosi risula manifesto il procedinento per la determinazione del valore del romelativo e delle rorrezioni dei lati.

In alcuni ea-i però is posibile ricorrere diretlamente alla ecfuazione (o alle equazioni) fra gli elementi lineari misurati, senza cesere

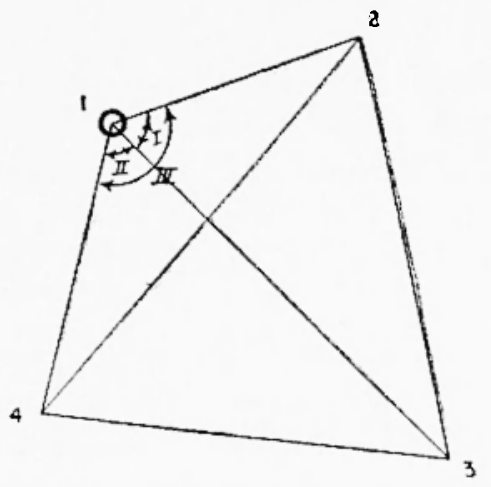

Ji!. $i$ obhligati al calcolo preventivo degli angroli ed alla conseguente compensizione.

A lale seopo consideriamo il raso di un quadrilatero di vertici $I$, 2. 3, 4 (fig. 7), ordinali in ordine cielico destrorso, i rui lati indichere. mo con le nolazioni (12), (23), (31), 1 fl) e le diaronali con le (13), 124). Fissando l'attenzione sul umo quatlunefue dei fuattro vertici, p. es. sul vertice 1, consideriamo i tre trianwoli aventi un angolo di vertice l (cioe i triangoli 123, 13.1, 124 ed a ciascumo di essi applichiamo il Teorema di Carnot in modo da ricavare i coseni degli angoli di ror. tice $1 \quad(2 \hat{\mathrm{l}} 3,3 \hat{\mathrm{l}} 4,2 \hat{\mathrm{l}} 4$ in funzione dei lati.

Poich:i :

$$
2 \hat{\imath}+=2 \widehat{1} 3+3 \hat{11}
$$

prendendo $i$ coseni dei due membri. siluppando il secondo membro ed esprimendo tutto in funzione dei soli co-eni. -i arriva con faciliti alla identita:

$$
\left.\cos ^{2}(2 \hat{1} 3)+\cos ^{2}(3 \hat{1} 4)+\cos ^{2}(2 \hat{1} 4)-2 \cos (\hat{2} 3) \cos (3 \widehat{1} 4) \cos (\hat{2} 4)-1=1\right) \text {. }
$$

Eliminando da questal le funzioni goniometriche introducendo i lati tramite i risultati ottenuti applicando ai triangoli accennati il Teoremat del coseno. si perviene allat seguente identita di Curmol:

$$
4(12)^{2}(13)^{-}(1.4)^{2}-\left\{(12)^{2}+(13)^{2}-(23)^{2}\right\}
$$




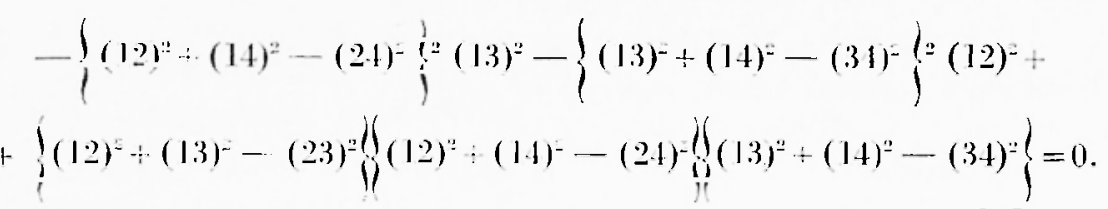

Lat [1] puó arthe exere serilla -ollo forma di delerminamle di lerzo ordine simmetrie ri-pello alla diagonale principale

$$
\left|\begin{array}{ccc}
1 & \cos (213) & \cos (214) \\
\cos (213) & 1 & \cdots=(314) \\
\cos (211) & 10=(3 ! 1) & 1
\end{array}\right|=0
$$

c. dia ruesia ponendo:

$$
A-2(12)(13)(14) A_{\mathrm{rs}}=\left\{(1 r)^{2}+(1 s)^{-}-(r s)^{2}: \frac{A}{2(\operatorname{lr})(1 s)}\right.
$$

-i perviene allia:

$$
\left|\begin{array}{ccc}
A & A_{2: 3} & A_{24} \\
A_{2: 3} & f & A_{: 4} \\
A_{2 !} & A_{: 1} & A
\end{array}\right|=0
$$

Whe at meno def faltore 4 reoincide con la $[2]$.

So-dituendo nella [2] e nelle equivalenti al posto delle lungheze (rs) i ri-ultali provenienti dille musure, si arriva alla delerminazione del residho di ossertazione $\lrcorner$ : allora, indicando con o (rs) le corre-

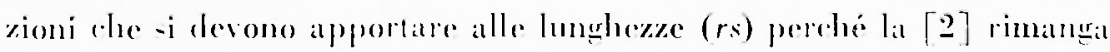

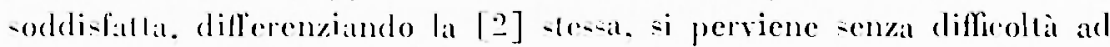
Huli erfuizione del lipo:

$$
M \delta(12) \cdots-1 \delta(23)-P \delta(34)-Q \delta(41)-R \delta(13) \div S \delta(24)=\lrcorner
$$

con fauile delerminazione dei coefferenti $M$. N. P. Q. R. S.

Indicando poi ron $K$ il correlativo, poichi $i$ :

$$
\begin{array}{lll}
\delta(12)=K . M, & \delta(23)=K . N . & \partial(3+)=K . P . \\
\delta(1+1)=K . O . & \partial(13)=K . R . & \delta(21)=K . S
\end{array}
$$

(…) risulterai delerminato dilliı:

$$
K=\Delta:\left(H^{2}+X^{2}+P^{2}-Q^{2}+R^{2}+S^{2}\right)
$$

con-entuentemente le [.5] delermineramno i valori pii probabili delle rorrezioni o(1:) are.

II "Coat and Geodelic Surrey" (U.S.) hat misurato le lunghezze dei lali a delle diagronali del fuidtrilatero: 
1) Lar Jumla, 2) Garden (ity. 3) (heyenne, -1) Imperial lrovando $\mathrm{i}$ senuenti valori. epprensi in minlia:

\begin{tabular}{|c|c|c|c|c|c|}
\hline \multicolumn{2}{|c|}{$I$ misura } & \multicolumn{2}{|c|}{$I I$ misurat } & \multicolumn{2}{|c|}{ dipleren=er } \\
\hline$(12)$ & 1.18 .53 .11 & $(21)$ & 1.18 .539 .5 & & \\
\hline$(3-1)$ & $17: 3.7-5.7$ & $(13)$ & 173.7 .171 & - & 1.1 \\
\hline$(.12)$ & $181.309 \%$ & $(2-1)$ & 181.309 .1 & + & 3 \\
\hline$(1.1)$ & 198.701 .3 & $(.11)$ & $198.7(190$ & + & 41 \\
\hline$(31)$ & .2 .7 .2890 & $(1: 3)$ & 2.27 .2368 & & 31 \\
\hline$(32)$ & $3(18.5) ! 1$ & $(23)$ & 308.52 .92 & - & 11. \\
\hline
\end{tabular}

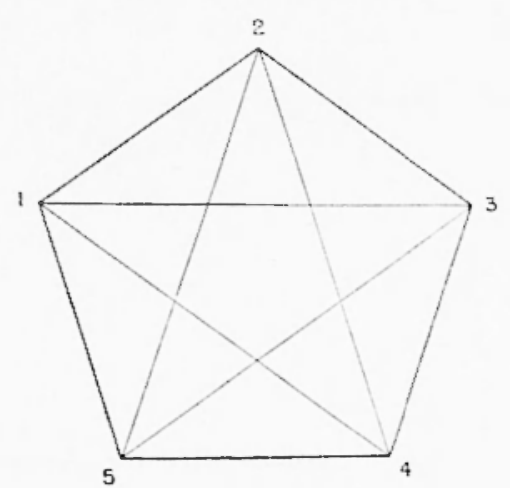

Fig. 8

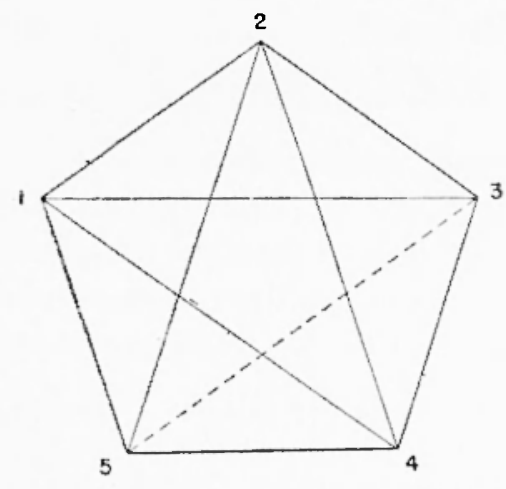

Fig. ")

I valori rompersati lisultano ordinatamente dei segunti imporli:

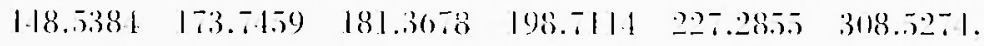

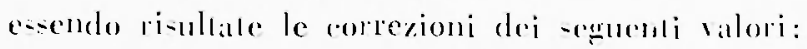

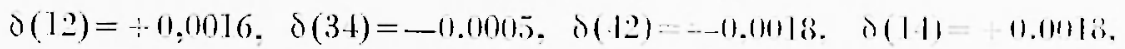
$\partial(31)=-10 .(11) 28 . \quad \partial(3.2)=10.11128$.

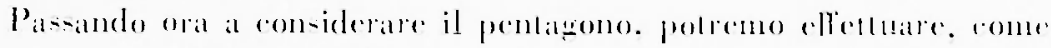

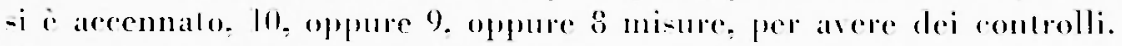

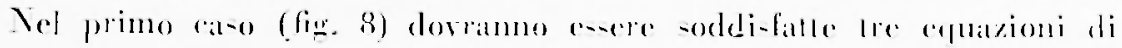
condizione del lipo [2] rieavate dai fuadrilateri rompleti (123)

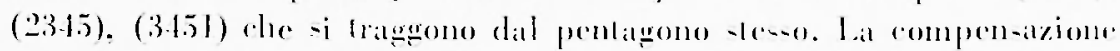
poi -i puo efícluare con sti ordinari procedimenti della Teoria dei minimi quadrali, per le owersazoni diente condizionale. 
Vel calso di g misure lpe e.s. lati o I diagonalis, -upponento fige. 9) ali non aver misuralo la diagromale (35). le equatzoni di romelizione sono due. entrambe del tipe $|2|$ rhe si ricalsano rontsideramdo i quadrilateri romple. li 112311 e 112151.

Mi-uramelo invere t lati e $\overline{-}$ diagenali, supponendo 1 fieg. 101 li mon aver misuralo il lalo 1.5, le expazioni di comelizione somo atreora dlue, definite lai fuatri-

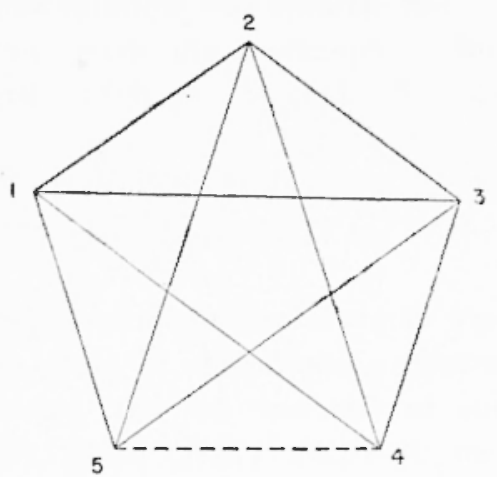

$\left[{ }^{\alpha x}.\right] 11$ lallo:i 112311. 1 12351.

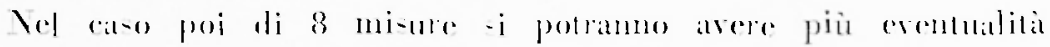

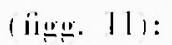

(1) Iati (12). (23). (34). (45). (51) diagonali (13). (25) (35)

b) idem

.) $x(12) \cdot(23),(31) \cdot(.51)$

)$\quad(13),(2.5) \cdot(2-1)$

") (13) $\cdot(35) \cdot(52) \cdot(2-1)$

(d) $)(12) \cdot(23) \cdot(51)$

$(52) .(21) \cdot(11) \cdot(13) \cdot(35)$

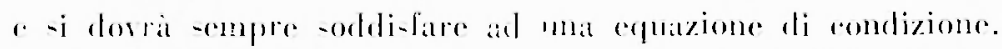

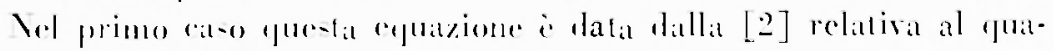

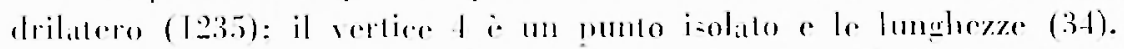
(1.i) 1100 -ono -owerette a romplensizione.

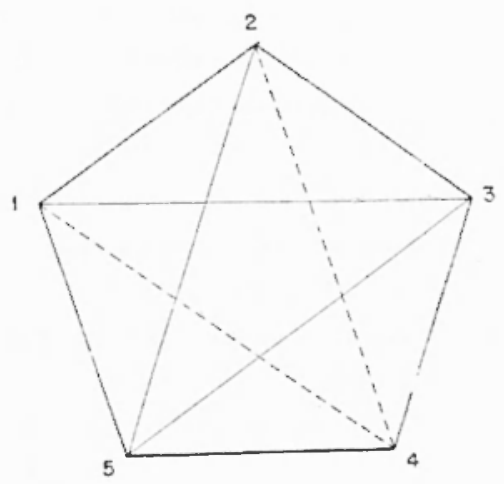

I:i

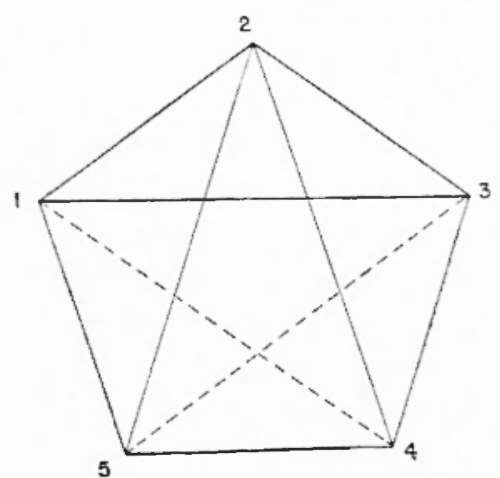

Fig. 1 l, 
Nel secondo caso entrano nella compensizione fute le misure esegruite. Ifequazione che deve essere sodelisatta 2 unal generalizzazione dell $_{a}[2]$. Essa si trae dalla identità groniometrical:

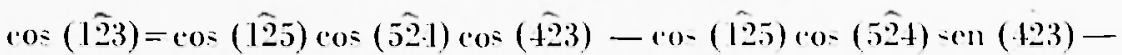
$-\cos (1 \hat{2} 5) \cos (+\sqrt[2]{3}) \operatorname{sen}(524)-\cos (5 \hat{2} 4) \cos (\sqrt{2} 3) \operatorname{sen}(1 \hat{2} 5)$

dopo di aver sostituito alle funzioni seno le funzioni coseno, razionalizzando e sostituendo al posto dei coseni le espressioni che si ottengono in funzione dei lati, ricorrendo al Teorema di Camot, per i triangoli (123). (125), (524). (423).

Negli ultimi due (at-i c). d). il vertice +l rimane i-olato. come nel

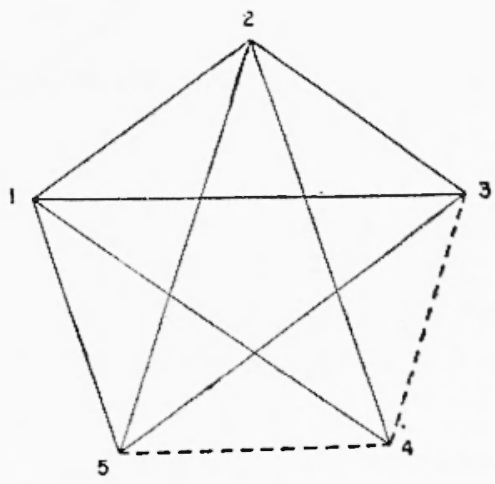

Fig. $11 \mathrm{c}$

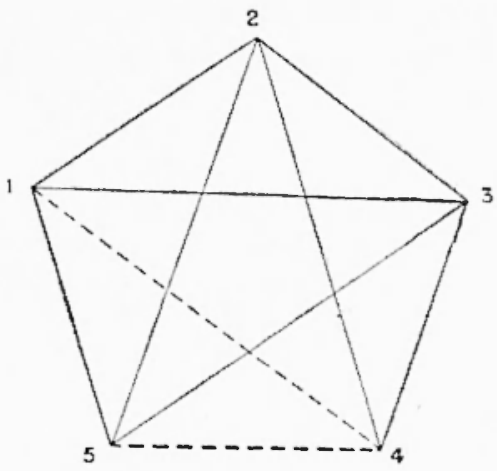

Fig. $11 \mathrm{~d}$

primo caso e le lunghezze da compensare sono solo quelle che defini-cono il quadrilatero completo (1235).

Si rede cosi dall'analisi falla rhe limitando ad 8 soltanto le misure, la conformazione piò idonea es quella data dal casoo b) per la quale tutti grli 8 elementi misurati entrano nella rompensazione.

In questo ordine di idee conviene anche con-iderare i ras riporlati nella fig. 12, dove con tratto rontinuo sono indicate le mintre offettuate, ma sostanzialmente il ratrionimento fatto non muta.

Col caso b) è slata fatta pre-ente la po-sibilita di unil generalizzazione dellat equazione [2], faceiamo ora presente che posiamo estenderla facilmente al caso di $n$ vertici $1,2,3, \ldots, n$ collenati come nel $\left(\begin{array}{cc}a s c o & b\end{array}\right)$.

Indieando ancora col simbolo ( $r s)$ le lunglezze dei segmenti in- 
dividualti dai vertici $r$ ed s. rhe potramo escere lati o diagranali, ponendo:

$$
I=2(12)(13)(14) \ldots(\ln ) \quad A_{\mathrm{r} z}=\left\{(1 r)^{2} \ldots(1 s)^{2}-(r s)^{2}\right\} \frac{A}{2(1 r)(1 s)}
$$

-i gimuge allal

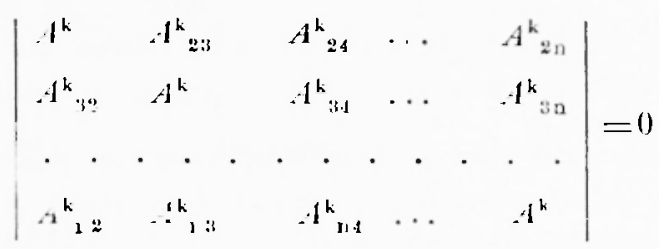

ron $l<\leq-3$. Per $n-4$ - i rieasal la [2].

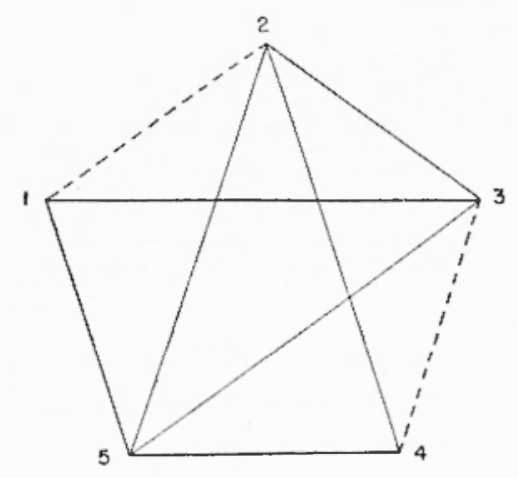

Fig. 12 a

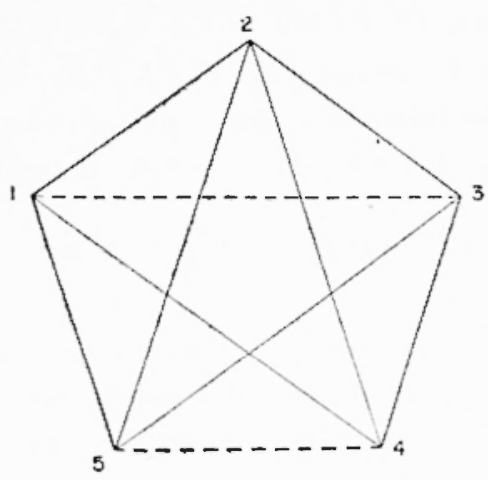

Fig. 12b

La $[6]$ C tata data da G. Candido nel 1935 in uno studio dal titolo "Generalizzazione di relazioni ela-iche per di-lanze di punti ". Lal [1] invece es sata scrita dal Briochi mel 185.5 (Nowr. Ann., pater. liz) solto la forma

$$
\left|\begin{array}{ccccc}
0 & 1 & 1 & 1 & 1 \\
1 & 11 & (12)^{2} & (13)^{2} & (14)^{2} \\
1 & (12)^{2} & 0 & (23)^{2} & (24)^{2} \\
1 & (13)^{2} & (23)^{2} & 0 & (31)^{2} \\
1 & (14)^{2} & (24)^{2} & (31)^{2} & 0
\end{array}\right|=0
$$

riportatia recentemente dal prof. Hart nella Nola ricordata nella inroduzionce. 


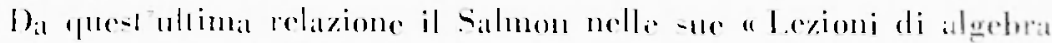

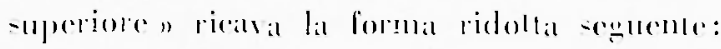

\begin{tabular}{|c|c|c|}
\hline$\ddot{2}(12)^{-2}$ & $(12)^{-1} \times(13)^{\circ}-(23)^{-}$ & $(12)+(1+1)-(2) 1)$ \\
\hline$(12)^{2}+(13)^{-}-(23)^{-}$ & $2(13)$ & $(13) \div(11)^{2}-(31)^{2}$ \\
\hline$(12)^{-}+(11)^{2}-(21)^{-}$ & $(13)^{*}+(1.11-(3)--$ & $2\{1\} ;$ \\
\hline
\end{tabular}

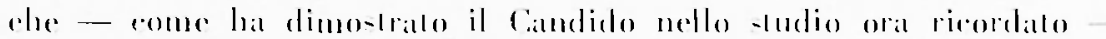

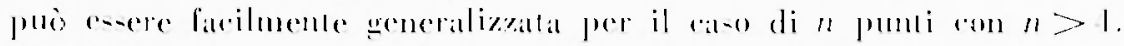

4. - 11 Radal puo escere anthe adoperato ron profitto nellat mi-

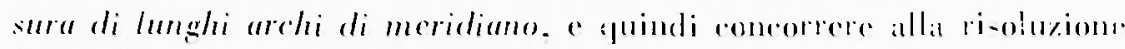

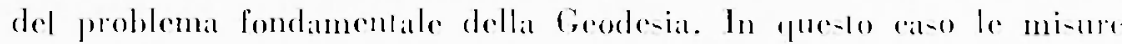

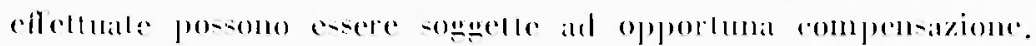

Co-i f. es se si dere misuma lo allineamento $P_{1} I_{1}$-udidisiden-

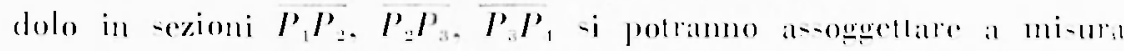

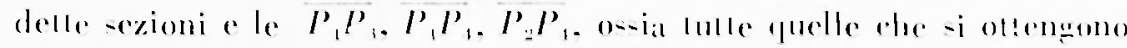

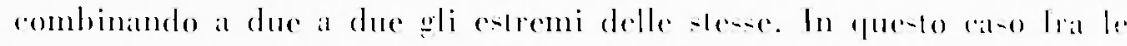

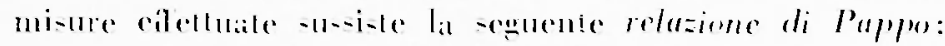

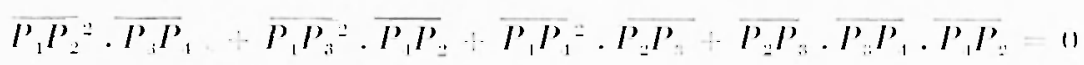

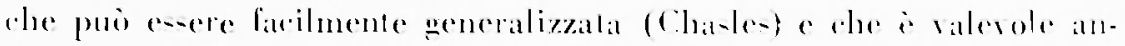

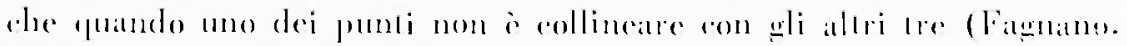
1.-1.3) con rhe i ottiene il rosi delto Teorrma di Stewarl.

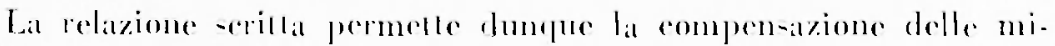

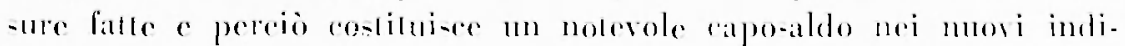
f'izzi nei melodi di rilevamento denli allineamenti.

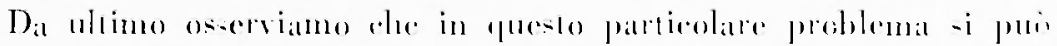

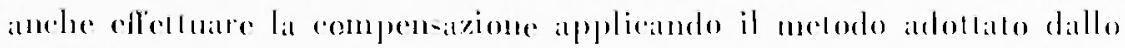

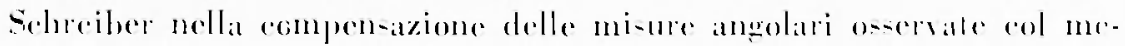
lodo delle combinazioni hinarie.

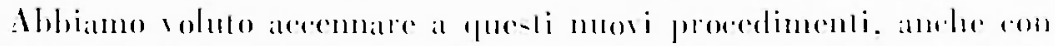

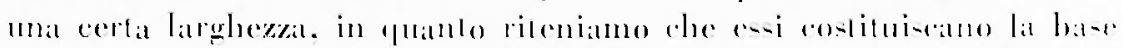

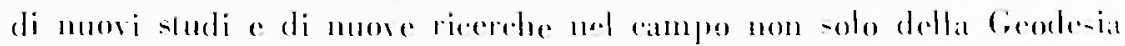

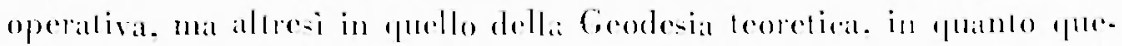
- li nuovi melodi allireano in maniera con-iderevole i rampi (p. a.

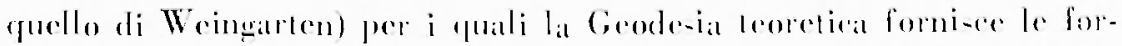
mule adalle per le ri-oluzioni dei vari poblemi geodelede fi rrovia-

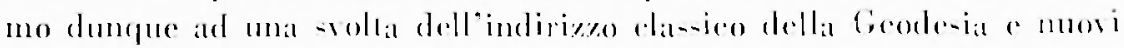


-lucli, move generalizazioni, -i impongono per poter ri-olvere i pro-

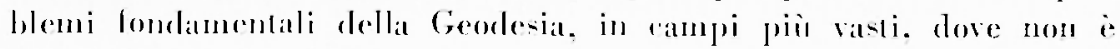

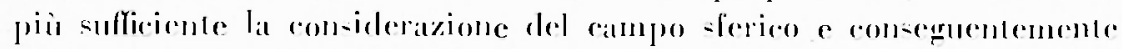
wli inclirizzi di Gall- -ulle superticie applicaluili.

Roma _- Istiluto di Cosodesia e Topografia della Facolla di Ingegneria 28 Mar=o 1919.

\section{IRI ASY VTO}

Jongeno dapprima illustrati bresemente i principh sui quali si basumo i procedimenti di mismazione delle dislanze con mezi reletrrici, poi angono messi in lace atemi importanli lan oni effelluati du-

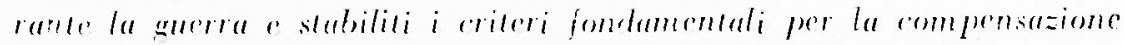
sella trilaterazionr. 